\title{
HEAT AND MASS TRANSFER EFFECT ON AN INFINITE VERTICAL PLATE IN THE PRESENCE OF HALL CURRENT AND THERMAL RADIATION WITH VARIABLE TEMPERATURE
}

\author{
L. MANJULA* \\ Department of Mathematics \\ SRM Institute of Science and Technology \\ Kattankulathur, Tamil Nadu 603203, INDIA \\ E-mail:manjulal@srmist.edu.in \\ R. MUTHUCUMARASWAMY \\ Department of Applied Mathematics \\ Sri Venkateswara College of Engineering, \\ Pennalur, Sriperumbudur Taluk 602117, INDIA
}

\begin{abstract}
MHD and radiated heat flow on a rotating system of an electrically conducting fluid in the presence of Hall current under the influence of variable temperature is studied analytically. An exact solution of a non-dimensional form of coupled partial differential equations is obtained by the technique of Laplace transform. The effect of temperature, velocity and concentration is analyzed for various parameters like the Hall parameter $(m)$, thermal radiation $(R)$, rotation parameter $(\Omega)$, Hartmann number $(M)$ and results are discussed in detail with the help of graphs. A mixed analysis of a rotating fluid with Hall current and thermal radiation plays a very essential role in the research area such as plasma physics, MHD generator, fluid drift sensor, cosmological and geophysical level, etc.
\end{abstract}

Keywords: MHD, Hall effects, radiation, vertical plate, variable temperature.

\section{Introduction}

The effect of a magnetic field on a fluid with natural convection plays a major role in electrolytes, liquid metal and ionized gases. The problem of an unsteady free convection flow is encountered in many natural phenomena and engineering applications with MHD analysis. An MHD flow with Hall current is encountered in engineering applications in MHD pumps, MHD bearings, etc. A free convection unsteady flow is of great interest in industrial fields such as fibre insulation, nuclear reactor, spacecraft design, geothermal system, etc. It also plays a very vital role in plasma physics and astrophysics. The Hall effect principle used in Hall effect joysticks, is used to control hypersonic flows, hydraulic valves, nuclear power reactors, brushless DC motors, calculate magnetic fields, build plasma actuator, and Hall current accelerators.

In a rotating system, Hall current on an accelerated plate or sheet was investigated by Pop. [1]. Muthucumaraswamy et al. [2] analyzed a magnetic flow with variable temperature on a vertical accelerated plate of a rotation system without mass diffusion. Deka [3] analyzed Hall current with MHD flow on an accelerated plate. Hall current and thermal characteristics effect in a rotating system in MHD flow was studied by Ghosh et al. [4]. Muthucumaraswamy and Jeyanthi [5] studied a rotating system effect of a first order chemical reaction and variable temperature with Hall current on an MHD flow. An exact solution of the radiation effect in the presence of variable temperature on a vertical moving plate with mass flux was studied by Muthucumaraswamy [6]. Thamizhsudar et al. [7] investigated an MHD free convection flow with Hall effect with combined MHT (Mass and Heat Transfer)

\footnotetext{
${ }^{*}$ To whom correspondence should be addressed
} 
in a rotating system. A hydro- magnetic natural convection flow with Hall effect in a spinning fluid was studied by Ram [8]. A hydromagnetic flow with Hall effect in a porous medium was studied by Gupta [9]. The inverse Laplace algorithm was studied by Hetnarski [10].

The objective of this paper is to study the thermal radiation on a magneto hydrodynamic fluid in the presence of a rotating system with Hall current on an isothermal vertical plate relative to uniform mass diffusion and variable temperature. The analytic solutions of dimensionless governing equations are expressed as a function of exponential and complement error function. Numerical results for concentration, temperature and fluid velocity profiles are represented by graphs which are executed by MATLAB.

\section{Mathematical formulation and solution}

Consider a viscous, incompressible transient flow of an electrically conducting fluid on an infinite vertical plate or sheet $\left(z^{\prime}=0\right)$. The plate motion is in upward direction along the $x^{\prime}$-axis and the $y^{\prime}$-axis is perpendicular to both the $x^{\prime}$-axis and the $z^{\prime}$-axis. Initially, $(t=0)$. The system and fluid rotate about the $z^{\prime}$ -axis with uniform angular velocity $-\boldsymbol{\Omega}^{\prime}$, which is normal to the sheet or plate. The fluid temperature - $T_{\infty}^{\prime}$ remains same at $t^{\prime} \leq 0$ and fluid concentration- $C_{\infty}^{\prime}$ also. At present work, the variable temperature effect on a transient flow of a viscous incompressible fluid on an infinite vertical plate with constant mass diffusion is considered. A uniform magnetic field $B_{0}$ acting parallel to the $z^{\prime}$-axis influences the fluid flow. Let $\left(u^{\prime}, v^{\prime}, 0\right)$ be a vector component of the velocity vector along the $x^{\prime}$-axis and $y^{\prime}$-axis respectively. At the same time, the system rotates along the $z^{\prime}$-axis in a constant angular velocity in anticlockwise direction. The motion of the plate started with velocity $u^{\prime}=u_{0}$ about the $x^{\prime}$-axis and the fluid temperature linearly raised with time $t^{\prime}$ and levels of concentration $\left(C_{w}^{\prime}\right)$ are also raised and kept constant. Since the transient flow depends upon $z^{\prime}$ and $t^{\prime}$, the fluid flow is maintained at same level apart from the plate. Let us assume that the transient flow is represented by a couple of partial differential equations. All physical parameters exempting pressure depend on $z^{\prime}$ and $t^{\prime}$ and also the flow is undisturbed far away from the plate. The governing equations of the present model with the above assumption are as follows:

$$
\begin{aligned}
& \frac{\partial u^{\prime}}{\partial t^{\prime}}=v \frac{\partial^{2} u^{\prime}}{\partial z^{\prime 2}} x+g \beta\left(T^{\prime}-T_{\infty}^{\prime}\right)-\left(\frac{u^{\prime}+m v^{\prime}}{1+m^{2}}\right) \frac{\sigma B_{0}^{2} \mu_{e}^{2}}{\rho}+2 \Omega_{z}^{\prime} v^{\prime}+g \beta^{*}\left(C^{\prime}-C_{\infty}^{\prime}\right), \\
& \frac{\partial v^{\prime}}{\partial t^{\prime}}=v \frac{\partial^{2} v^{\prime}}{\partial z^{\prime 2}}-\left(\frac{v^{\prime}-m u^{\prime}}{1+m^{2}}\right) \frac{\sigma B_{0}{ }^{2} \mu_{e}^{2}}{\rho}-2 \Omega_{z} u^{\prime} \\
& \frac{\partial T^{\prime}}{\partial t^{\prime}}+\frac{1}{\rho C_{p}} \frac{\partial q_{r}}{\partial z^{\prime}}=A \frac{\partial^{2} T^{\prime}}{\partial z^{\prime 2}}, \quad \text { where } \quad A=\frac{k}{\rho C_{p}}, \\
& \frac{\partial C^{\prime}}{\partial t^{\prime}}=D \frac{\partial^{2} C^{\prime}}{\partial z^{\prime 2}}-K_{t}\left(C^{\prime}-C_{\infty}^{\prime}\right) .
\end{aligned}
$$

The axial velocity component is denoted by $u^{\prime}$ along the $x^{\prime}$-axis and the transverse velocity component is denoted by $v^{\prime}$ along the $y^{\prime}$-axis of the flow. When the angular velocity $\Omega$ is equal to $\frac{m M^{2}}{1+m^{2}}$, the velocity reaches its peak value and the transverse velocity decreases to zero.

The initial value and boundary value are given as below: 


$$
\begin{aligned}
& \text { for all } \quad z^{\prime}, \quad \text { and } \quad t^{\prime} \leq 0, \quad T^{\prime}=T_{\infty}^{\prime}, \quad u^{\prime}=v^{\prime}=0, \quad \text { and } \quad C^{\prime}=C_{\infty}^{\prime}, \\
& \text { for all } \quad t^{\prime}>0, \quad T^{\prime}=T_{w}^{\prime}, \quad u^{\prime}=u_{0}, \quad v^{\prime}=0, \quad C^{\prime}=C_{w}^{\prime} \quad \text { at } \quad z^{\prime}=0, \\
& T^{\prime} \rightarrow T_{\infty}^{\prime}, \quad u \rightarrow 0, \quad v \rightarrow 0, \quad C^{\prime} \rightarrow C_{\infty}^{\prime} \quad \text { as } \quad z \rightarrow \infty .
\end{aligned}
$$

Thin gray gas' local radiant expression is as noted below:

$$
\frac{\partial q_{r}}{\partial z^{\prime}}=-4 a^{*}\left(T_{\infty}^{\prime 4}-T^{\prime 4}\right) \sigma^{*}
$$

where $\sigma^{*}$ denotes the Stefan-Boltzmann constant and $a^{*}$ represents the coefficient of heat absorption. In the boundary layer, assume that the fluid difference in temperature is sufficiently small. Using Taylor's expansion at $T_{\infty}^{\prime}$, we get:

$$
T^{\prime 4}+3 T_{\infty}^{\prime 4}=4 T_{\infty}^{\prime 3} T^{\prime}
$$

Using (2.7) and (2.8) in Equation (2.3), we obtain

$$
\rho C_{p} \frac{\partial T^{\prime}}{\partial t^{\prime}}=k \frac{\partial^{2} T^{\prime}}{\partial z^{\prime 2}}+16 a^{*} \sigma^{*} T_{\infty}^{\prime 4}\left(T_{\infty}^{\prime}-T_{\infty}^{\prime}\right)+k \frac{\partial^{2} T^{\prime}}{\partial z^{\prime 2}}
$$

Non-dimensional quantities are introduced by Eq.(2.10) and used in Eqs (2.1), (2.2), (2.4) and (2.9)

$$
\begin{aligned}
& u=\frac{u^{\prime}}{u_{0}}, \quad v=\frac{v^{\prime}}{v_{0}}, \quad t=\frac{t^{\prime} u_{0}^{2}}{v}, \quad z=z^{\prime} \frac{u_{0}^{2}}{v^{2}}, \quad \Omega=\Omega_{z}^{\prime} \frac{v}{u_{0}^{2}}, \quad \operatorname{Pr}=\frac{\mu C_{p}}{k} \\
& G r=\frac{v g \beta\left(T_{w}^{\prime}-T_{\infty}^{\prime}\right)}{u_{0}^{3}}, \quad G c=\frac{\operatorname{vg} \beta^{*}\left(C_{w}^{\prime}-C_{\infty}^{\prime}\right)}{u_{0}^{3}}, \quad R=\frac{\left.16 \sigma^{\bullet} a^{*} T_{\infty}^{\prime 3} v^{2}\right)}{k \operatorname{Pr} u_{0}^{2}} \\
& M^{2}=\frac{\sigma \mu_{e}{ }^{2} B_{0}^{2} v}{2 \rho u_{0}^{2}}, \quad \theta=\frac{T^{\prime}-T_{\infty}^{\prime}}{T_{w}^{\prime}-T_{\infty}^{\prime}}, \quad S_{C}=\frac{v}{D}, \quad K=K_{l} \frac{v}{u_{0}^{2}}, \quad C=\frac{C^{\prime}-C_{\infty}^{\prime}}{C_{w}^{\prime}-C_{\infty}^{\prime}} .
\end{aligned}
$$

The governing equations are reduced to a non-dimension form

$$
\begin{aligned}
& \frac{\partial u}{\partial t}=2 \Omega v+G r \theta+\frac{\partial^{2} u}{\partial Z^{2}}+G c C-M^{2}\left(\frac{u+m v}{1+m^{2}}\right), \\
& \frac{\partial v}{\partial t}+2 \Omega u=\frac{\partial^{2} v}{\partial z}+M^{2}\left(\frac{m u-v}{1+m^{2}}\right), \\
& \frac{\partial \theta}{\partial t}+R \theta=\frac{1}{\operatorname{Pr}}\left(\frac{\partial^{2} \theta}{\partial z^{2}}\right)
\end{aligned}
$$




$$
\frac{\partial C}{\partial t}+K C=\frac{1}{S_{C}}\left(\frac{\partial^{2} C}{\partial z^{2}}\right)
$$

with initial and boundary conditions

at $t \leq 0$

$$
u=0, \quad v=0, \quad \theta=0, \quad C=0 \quad \text { for all } z,
$$

when $t>0$,

$$
\begin{aligned}
& u=1, \quad v=0, \quad \theta=t, \quad C=1 \quad \text { at } \quad z=0, \\
& u \rightarrow 0, \quad \theta \rightarrow 0, \quad v \rightarrow 0, \quad C \rightarrow 0 \quad \text { as } \quad z \rightarrow \infty .
\end{aligned}
$$

Combining the governing Eq.(2.11) and (2.12), we get

$$
\frac{\partial F}{\partial t}=\frac{\partial^{2} F}{\partial z^{2}}-a F+G r \theta+G c C
$$

where

$$
\begin{aligned}
& a=\frac{2 M^{2}}{1+m^{2}}+2 i\left(\Omega-\frac{M^{2} m}{1+m^{2}}\right) \quad \text { and } F=u+i v, \\
& \frac{\partial \theta}{\partial t}+R \theta=\frac{1}{\operatorname{Pr}}\left(\frac{\partial^{2} \theta}{\partial z^{2}}\right), \\
& \frac{\partial C}{\partial t}+K C=\frac{1}{S c}\left(\frac{\partial^{2} C}{\partial z^{2}}\right) .
\end{aligned}
$$

The transverse velocity vanishes when the rotation parameter $\Omega$ is $\frac{m M^{2}}{1+m^{2}}$.

Conditions (2.15) transform into

at $t \leq 0$

$$
F=0, \quad \theta=0, \quad C=0 \quad \text { for all } \quad z,
$$

when $t>0$

$$
\begin{aligned}
& F=1, \quad \theta=t, \quad C=1 \quad \text { at } \quad z=0, \\
& \theta \rightarrow 0, \quad F \rightarrow 0, \quad C \rightarrow 0 \quad \text { as } \quad z \rightarrow \infty
\end{aligned}
$$




\section{Discussion of exact solution} transform:

Exact solutions of the fluid velocity, temperature and concentration are obtained by the inverse Laplace

$$
\begin{aligned}
& \theta(z, t)=\frac{1}{2}\left[t\left(E_{3} \operatorname{erfc}(\eta \sqrt{\operatorname{Pr}}-\sqrt{R t})+E_{4} \operatorname{erfc}(\eta \sqrt{\operatorname{Pr}}+\sqrt{R t})\right)+\right. \\
& \left.+\frac{\eta \sqrt{P_{r} t}}{\sqrt{R}}\left(E_{3} \operatorname{erfc}(\eta \sqrt{\operatorname{Pr}}-\sqrt{R t})+E_{4} \operatorname{erfc}(\eta \sqrt{\operatorname{Pr}}+\sqrt{R t})\right)\right] \text {, } \\
& C(z, t)=\frac{1}{2}\left[E_{1} \operatorname{erfc}\left(\eta \sqrt{S_{c}}-\sqrt{K t}\right)+E_{2} \operatorname{erfc}\left(\eta \sqrt{S_{c}}+\sqrt{K t}\right)\right], \\
& F(z, t)=\left(1-\frac{A}{\gamma}-B\right)\left[E_{5} \operatorname{erfc}(\eta-\sqrt{a t})+E_{6} \operatorname{erfc}(\eta+\sqrt{a t})\right]+ \\
& +\frac{A}{2 \gamma}\left\{\operatorname { e x p } ( \gamma t ) \left[E_{7} \operatorname{erfc}(\eta-\sqrt{(a+\gamma) t})+E_{8} \operatorname{erfc}(\eta+\sqrt{(a+\gamma) t})+\right.\right. \\
& \left.-\left(E_{9} \operatorname{erfc}(\eta \sqrt{\operatorname{Pr}}-\sqrt{(R+\gamma) t})+E_{10} \operatorname{erfc}(\eta \sqrt{\operatorname{Pr}}+\sqrt{(R+\gamma) t})\right)\right]+ \\
& +E_{3} \operatorname{erfc}(\eta \sqrt{\operatorname{Pr}}-\sqrt{R t})+E_{4} \operatorname{erfc}(\eta \sqrt{\operatorname{Pr}}+\sqrt{R t})+ \\
& -\gamma t\left(E_{5} \operatorname{erfc}(\eta-\sqrt{a t})+E_{6} \operatorname{erfc}(\eta+\sqrt{a t})\right)+ \\
& +\gamma \frac{\eta \sqrt{t}}{\sqrt{a}}\left(E_{5} \operatorname{erfc}(\eta-\sqrt{a t})+E_{6} \operatorname{erfc}(\eta+\sqrt{a t})\right)+ \\
& -\gamma t\left[E_{3} \operatorname{erfc}(\eta \sqrt{\operatorname{Pr}}-\sqrt{R t})+E_{4} \operatorname{erfc}(\eta \sqrt{\operatorname{Pr}}+\sqrt{R t})\right]+ \\
& \left.+\frac{\gamma \eta \sqrt{\operatorname{Pr} t}}{\sqrt{R}}\left[E_{3} \operatorname{erfc}(\eta \sqrt{\operatorname{Pr}}-\sqrt{R t})-E_{4} \operatorname{erfc}(\eta \sqrt{\operatorname{Pr}}+\sqrt{R t})\right]\right\}+ \\
& +\frac{B}{2}\left\{\operatorname { e x p } ( \alpha t ) \left[E_{13} \operatorname{erfc}(\eta-\sqrt{(a+\alpha) t})+E_{14} \operatorname{erfc}(\eta+\sqrt{(a+\alpha) t})+\right.\right. \\
& \left.-\left(E_{11} \operatorname{erfc}(\eta \sqrt{S c}-\sqrt{(K+\alpha) t})+E_{12} \operatorname{erfc}(\eta \sqrt{S c}+\sqrt{(K+\alpha) t})\right)\right] \\
& \left.+E_{1} \operatorname{erfc}(\eta \sqrt{S c}-\sqrt{K t})-E_{2} \operatorname{erfc}(\eta \sqrt{S c}+\sqrt{K t})\right\}
\end{aligned}
$$

where

$$
\begin{aligned}
& \eta=\frac{Z}{2 \sqrt{t}}, \quad \alpha=\frac{a-K S c}{(S c-1)}, \quad \gamma=\frac{a-R \operatorname{Pr}}{(\operatorname{Pr}-1)}, \quad A=\frac{G_{r}}{\gamma(\operatorname{Pr}-1)}, \quad B=\frac{G_{c}}{\alpha\left(S_{c}-1\right)}, \\
& E_{1}=\exp \left(-2 \eta \sqrt{S_{c} K t}\right), \quad E_{2}=\exp \left(2 \eta \sqrt{S_{c} K t}\right), \quad E_{3}=\exp (-2 \eta \sqrt{R \operatorname{Pr} t}), \\
& E_{4}=\exp (2 \eta \sqrt{R \operatorname{Pr} t}), \quad E_{5}=\exp (-2 \eta \sqrt{a t}), \quad E_{6}=\exp (2 \eta \sqrt{a t}),
\end{aligned}
$$




$$
\begin{aligned}
& E_{7}=\exp (-2 \eta \sqrt{(a+\gamma) t}), \quad E_{8}=\exp (2 \eta \sqrt{(a+\gamma) t}), \quad E_{9}=\exp (-2 \eta \sqrt{\operatorname{Pr}(R+\gamma) t}), \\
& E_{10}=\exp (2 \eta \sqrt{\operatorname{Pr}(R+\gamma) t}), \quad E_{11}=\exp (-2 \eta \sqrt{S c(K+\alpha) t}), \\
& E_{12}=\exp (2 \eta \sqrt{S c(K+\alpha) t}), \quad E_{13}=\exp (-2 \eta \sqrt{(a+\alpha) t}), \\
& E_{14}=\exp (2 \eta \sqrt{(a+\alpha) t}) .
\end{aligned}
$$

\section{Results and discussion}

The impact of physical parameters such as the magnetic parameter $(M)$, Hall parameter $(m)$, radiation parameter $(R)$, chemical reaction parameter $(k)$, rotation parameter $(\boldsymbol{\Omega})$ and the non-dimensional number such as the Prandtl number - Pr, Schmidt number - $S_{c}$ and thermal Grashof number - $G_{r}$ on temperature $(\theta)$, concentration $(C)$ and axial velocity field $(F)$ is studied for different numerical values. The temperature profiles for different values of the parameters are described in Figs 1-4. For $t=2$ and different values of $R$ and $\operatorname{Pr}$, the distribution of temperature is shown in Fig.1. In Fig.2., the temperature field is described for different values of $\operatorname{Pr}$ (air and water) with different values of radiation and time. It is noted that the temperature increases as the value of time increases. This trend reverses with respect to radiation and the Prandtl number. Figure 3 shows the temperature distribution for $t=1,2,3$, corresponding to $R=0.2,0.5,1$ and for constant value of $\operatorname{Pr}=0.71$, the temperature increases. In Fig.4, for $t$ and $\operatorname{Pr}$ constant $(2$ and 0.71 , respectively), for increasing values of $R$, the temperature decreases.

Figures 5 and 6 show that the concentration boundary rises with the fall of $S c$ (Schmidt number) and $k$ (reaction parameter). Concentration in the presence of $S c=2.01$ (ethyl benzene) is less when compared to $S c=0.16$, (hydrogen) and $S c=0.6$ (water vapour) (see Fig.5) with various values of $k=0.2,2,5$ and $t=2$. Figure 6 shows that the concentration boundary falls with an increase in the chemical reaction parameter $(k)$ at $S c=2.01$ and $t=2$.

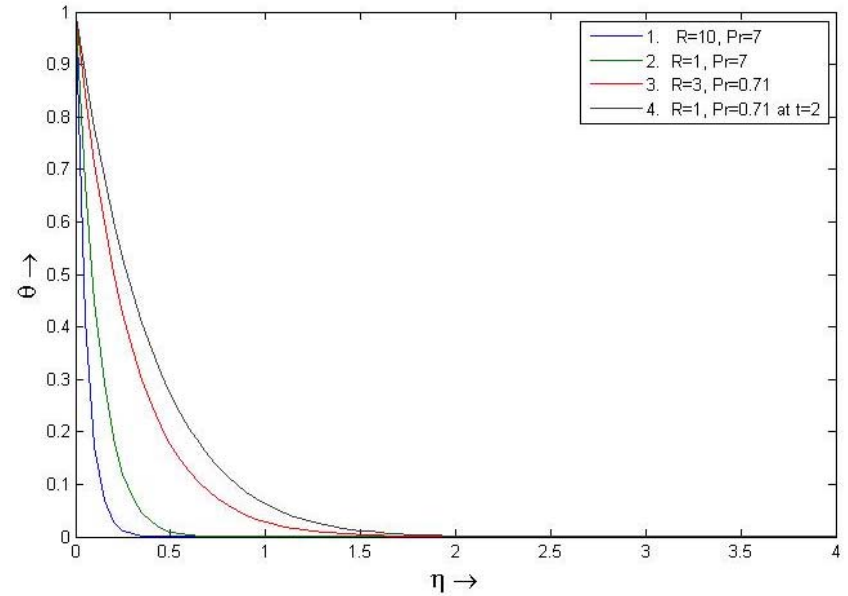

Fig.1. Temperature profile for different values of $\operatorname{Pr}$ and $R$ and $t=2$.

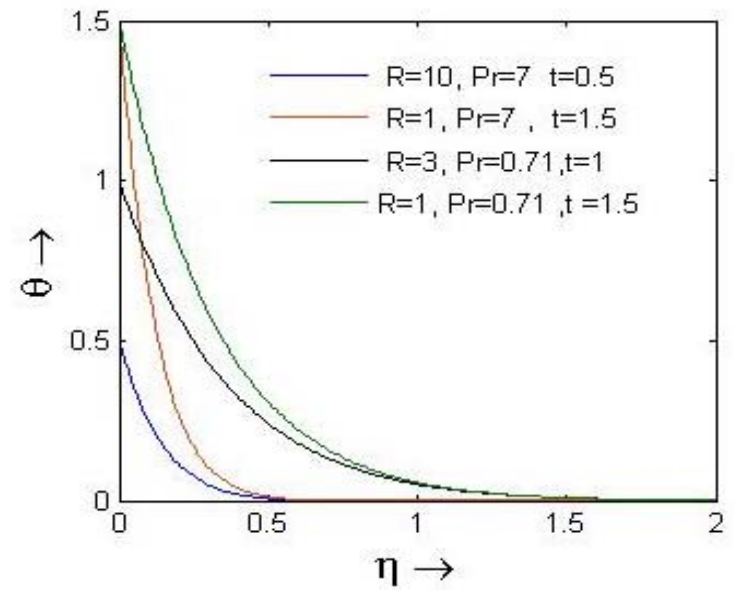

Fig.2. Temperature profile for different values of $R, \operatorname{Pr}$ and $t$. 


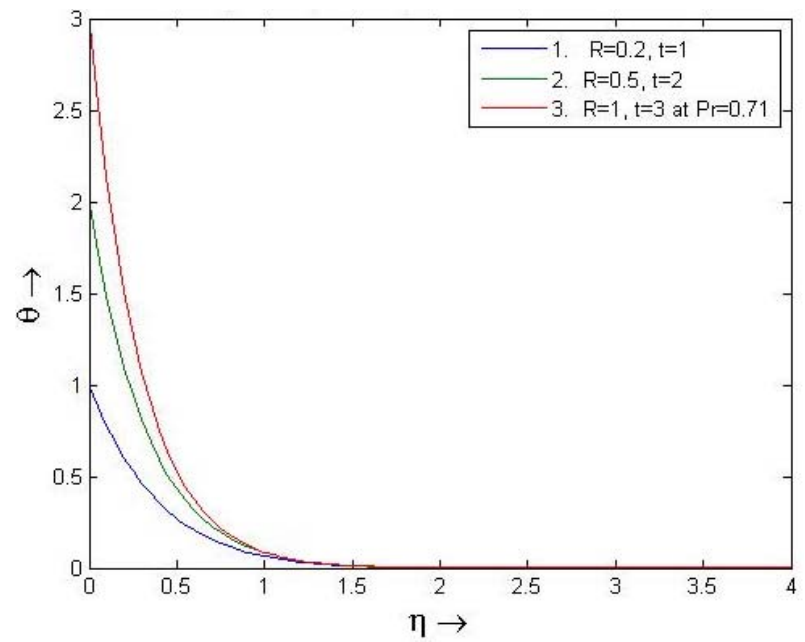

Fig.3. Temperature profile for different values of $R$ and $t$ for $\operatorname{Pr}=0.71$

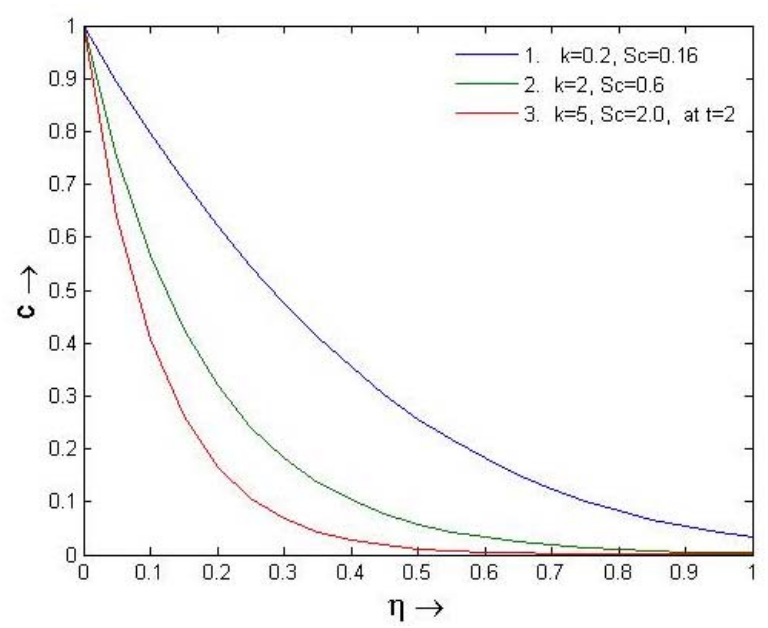

Fig.5. Concentration profiles for different values of $S c$ and $k$.

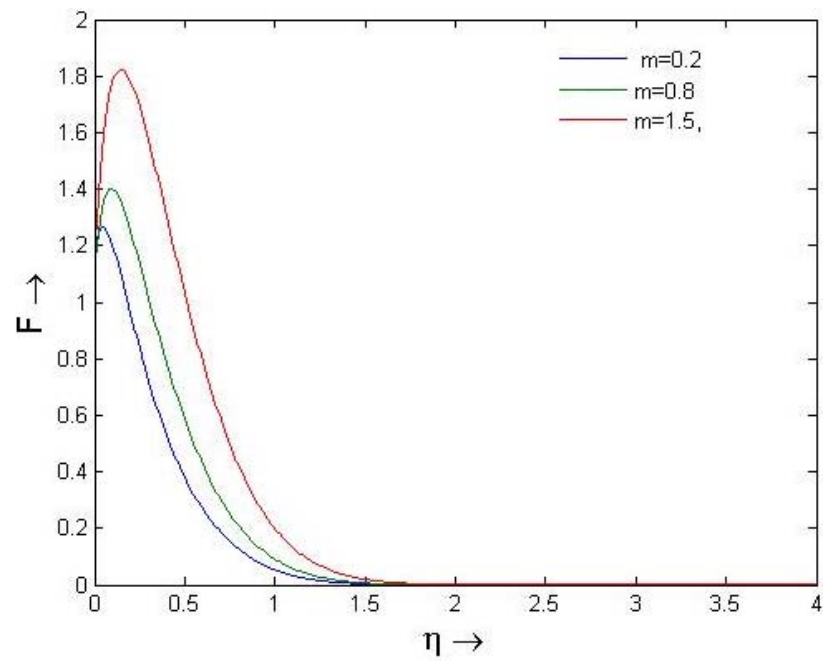

Fig.7. Velocity profiles for different values of the Hall parameter.

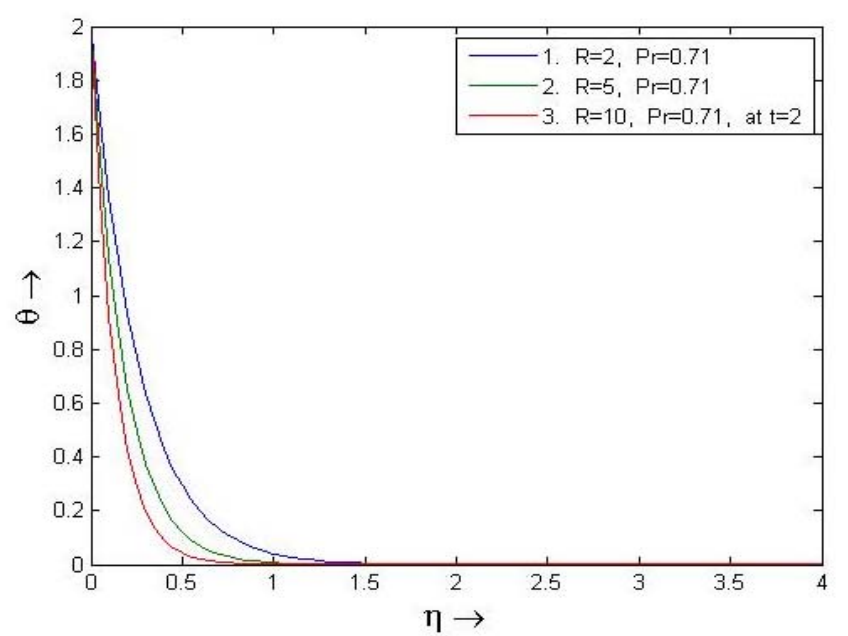

Fig.4. Temperature profile for different values of $R$.

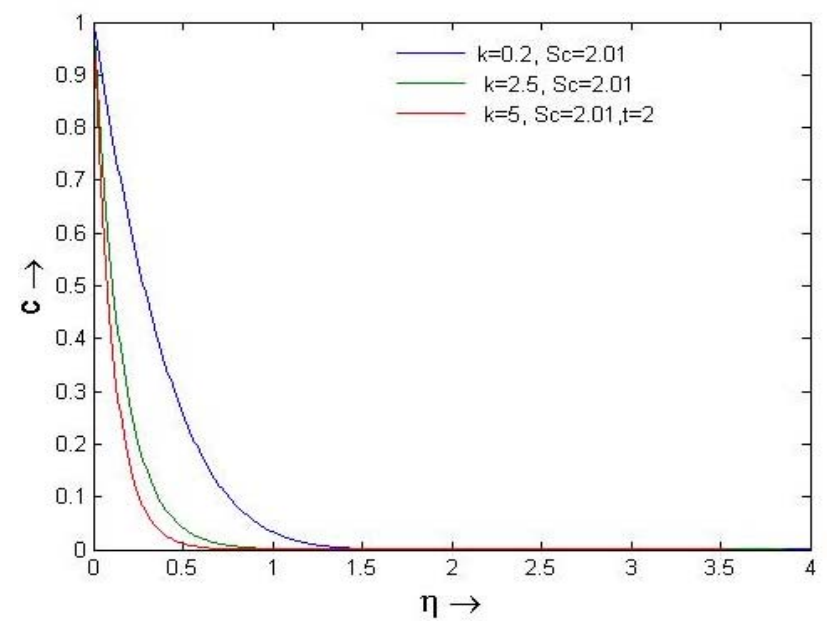

Fig.6. Concentration profiles for different values of $k$ for $S c=2.01$

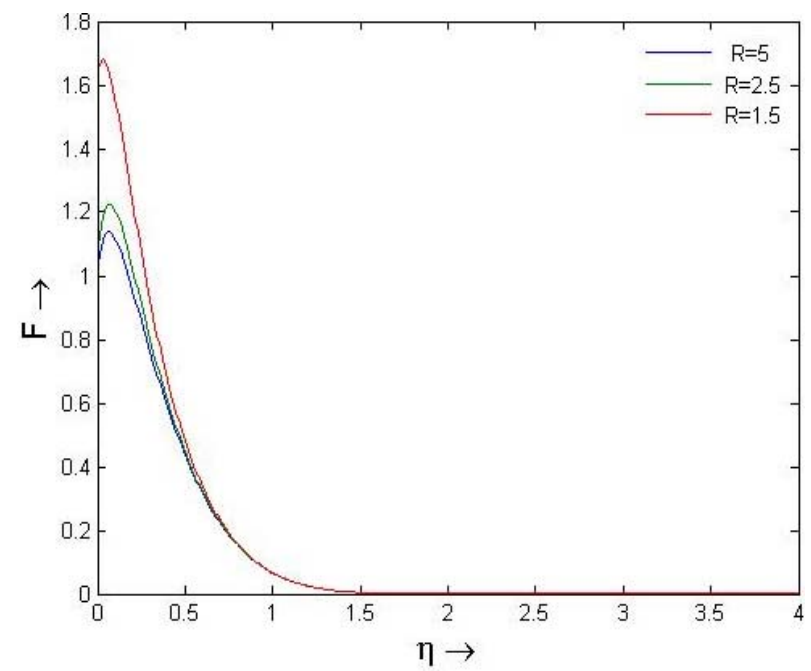

Fig.8. Velocity profiles for different values of radiation. 
The profiles of auxiliary velocity $F$ with various parameters $m, R, M, G r, G c, K, S c$, are illustrated in Figs 7-12. From Fig.7., it is clear that velocity increases with the increase in the Hall parameter $(m)$; where $S c=2.01, M=2, \operatorname{Pr}=7, k=0.2, G r=5, G c=10, R=2, \Omega=2$ and $t=2$. For different values of radiation $(R)$ when $m=0.5, S c=2.01, M=2, \operatorname{Pr}=7, k=0.2, \Omega=2, G r=5$, $G c=10$ and $t=2$, the velocity distribution is shown in Fig.8. It is evident that the velocity $F$ falls when $R$ is increased. Figure 9 shows that as the magnetic parameter $(M)$ increases, the velocity decreases when $\Omega=2, \operatorname{Pr}=7, k=0.2, G r=5, G c=10, R=2, t=2$ and $m=0.5$. We can observe that in Fig.10., the velocity $F$ increases with an increase in the thermal Grashof number $(G r)$ and mass Grashof number $(G c)$.

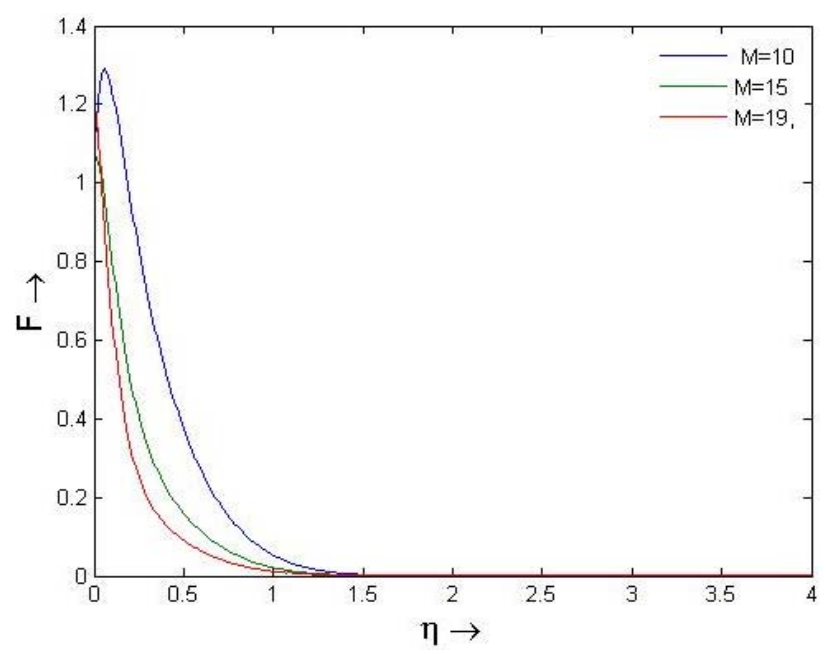

Fig.9. Velocity profiles for different values of the Hartmann number.

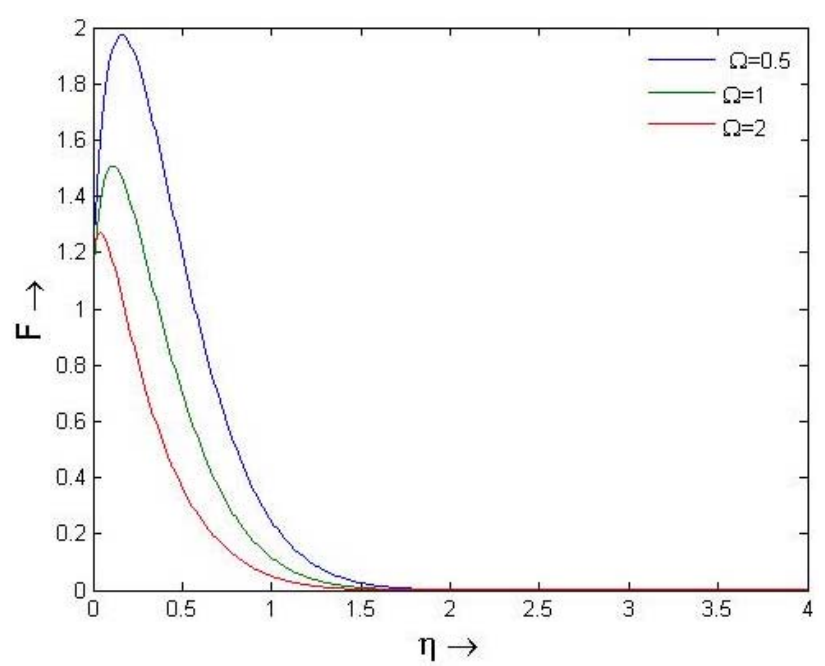

Fig.11. Velocity profiles for different values of the rotation parameter.

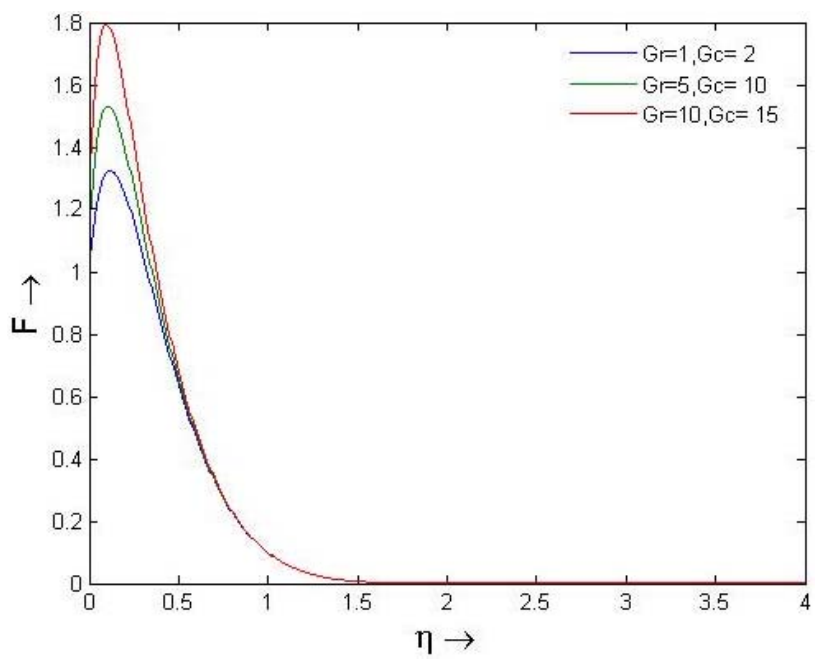

Fig.10. Velocity profiles for different values of $G r$ and $G c$.

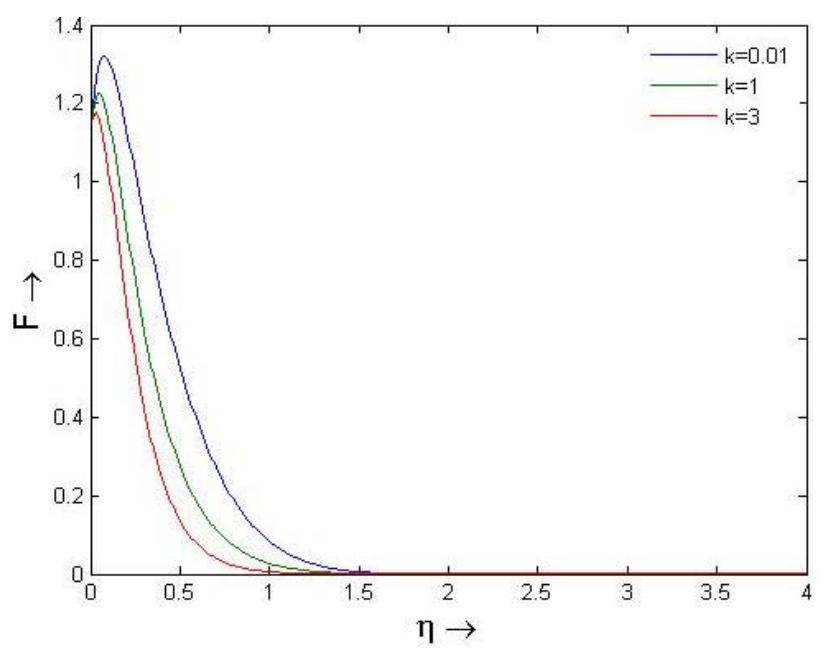

Fig.12. Velocity profiles for different values of chemical reaction. 
Figure 11 shows that for different values of the rotation parameter $\Omega$ when $\operatorname{Pr}=7, k=0.2, G r=5, G c=10$, $R=2, t=2, m=0.5$ and $S c=2.01$, it is noticed that the velocity field goes up as the rotation parameter values drop down. One can also see in Fig.12. that velocity falls when the chemical reaction value goes up.

\section{Conclusion}

Hall effect and radiation effect have been studied under theoretical solution of heat and mass transfer in the presence of a rotating fluid with variable temperature. The effect of different parameters on velocity, concentration and temperature field is presented graphically.

The conclusions of the study are follows:

- The temperature distribution increases with an increase of time and decrease of Pr - Prandtl number and radiation parameter $(R)$.

- The velocity profiles decreas with a decrease in $G r, G c$, and Hall parameter $(m)$.

- Velocity increases with a decrease in the rotation parameter, chemical reaction parameter, magnetic parameter $(M)$ and radiation parameter $(R)$.

- The concentration field decreases with a decrease of $S c$ and $k$.

\section{Nomenclature}

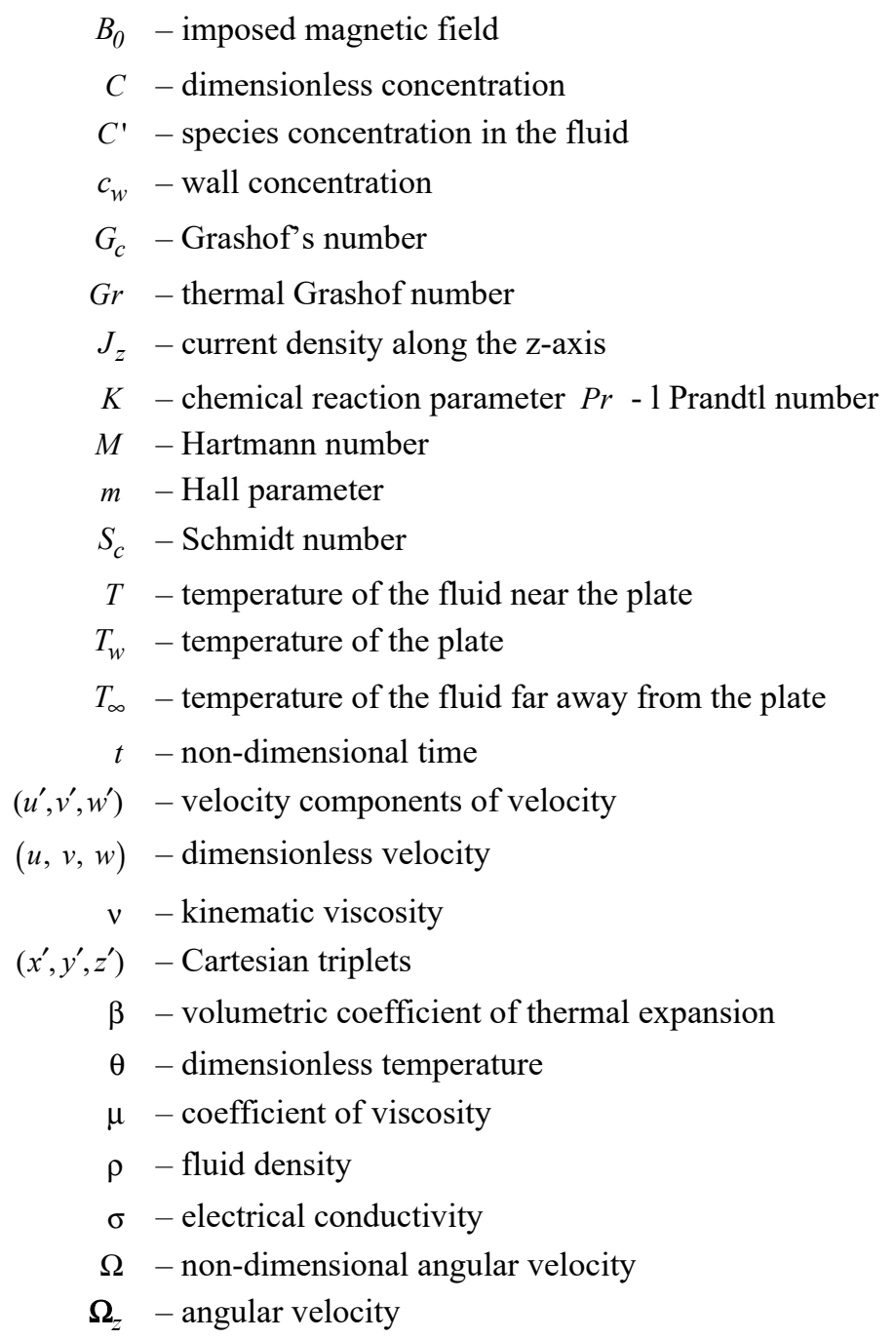




\section{References}

[1] Pop. I. (1971): The effect of Hall currents on hydromagnetic flow near an accelerated plate.- Journal of Mathematics and Physical Sciences. vol.5, pp.375-379.

[2] Muthucumaraswamy R., Tina Lal and Ranganayakulu D. (2011): Rotation effects on MHD flow past a vertical accelerated plate with variable temperature and uniform mass diffusion.- Annlals of Faculty of Engineering Hunedoara, vol.9, No.1, pp.228-239.

[3] Deka R.K. (2008): Hall effects on MHD flow past an accelerated plate, Theoretic.- Appl. Mech., vol.35, pp.333-346.

[4] Ghosh S.K., Anwar Bég O. and Narahari M. (2009): Hall effects on MHD flow in a rotating system with heat transfer characteristics.- Meccanica, vol.44, Article number: 741.

[5] Muthucumarswamy R. and Jeyanthi L. (2014): Hall Effects on MHD flow past an infinite vertical plate in the presence of rotating fluid of variable temperature and uniform mass diffusion with first order chemical reaction.- International Journal of Applied Engineering Research, vol.9, pp.26259-26271.

[6] Muthucumaraswamy R. (2010): Exact solution of thermal radiation on vertical oscillating plate with variable temperature and mass flux.-Appl. Mech., vol.37, No.1, pp.1-15.

[7] Thamizhsudar M., Muthucumaraswamy R., Pandurangan J. (2015): Hall effects and rotation effects on MHD flow past an exponentially accelerated vertical plate with combined heat and mass transfer effects.- International Journal of Applied Mechanics and Engineering, vol.20, No.3, pp.605-616

[8] Ram P.C. (1995): Hall effect on hydro magnetic convection flow in a rotating fluid.- Astrophysics and Space Science, vol.158, No.2, pp.189-195.

[9] Gupta S. (1975): Hydromagnetic flow past a porous flat plate with Hall effect.- Acta Mechanica, vol.22, pp.281-28.

[10] Hetnarski R.B. (1975): An algorithm for generating some inverse Laplace transforms of exponential form.- ZAMP, vol.26, pp.249-253.

Received: April 1, 2021

Revised: July 15, 2021 